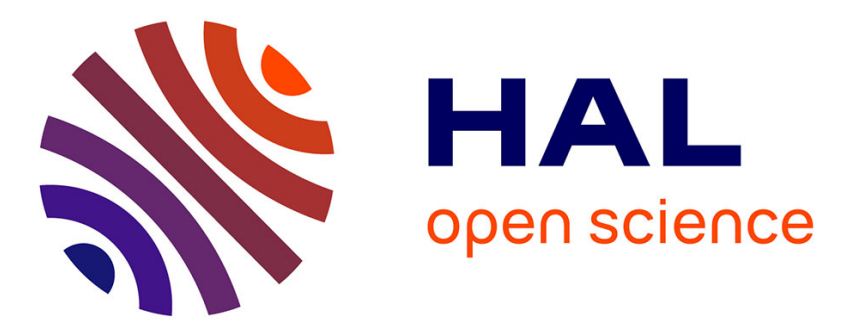

\title{
Molecular dynamics simulations for CO2 spectra. IV. Collisional line-mixing in infrared and Raman bands
}

Julien Lamouroux, Jean-Michel Hartmann, Ha Tran, Bruno Lavorel, Marcel Snels, Stephania Stefani, Giuseppe Piccioni

\section{To cite this version:}

Julien Lamouroux, Jean-Michel Hartmann, Ha Tran, Bruno Lavorel, Marcel Snels, et al.. Molecular dynamics simulations for CO2 spectra. IV. Collisional line-mixing in infrared and Raman bands. Journal of Chemical Physics, 2013, 138, pp.244310. 10.1063/1.4811518 . hal-00840845

\section{HAL Id: hal-00840845 https://hal.science/hal-00840845}

Submitted on 8 Jul 2013

HAL is a multi-disciplinary open access archive for the deposit and dissemination of scientific research documents, whether they are published or not. The documents may come from teaching and research institutions in France or abroad, or from public or private research centers.
L'archive ouverte pluridisciplinaire HAL, est destinée au dépôt et à la diffusion de documents scientifiques de niveau recherche, publiés ou non, émanant des établissements d'enseignement et de recherche français ou étrangers, des laboratoires publics ou privés. 


\title{
Molecular dynamics simulations for $\mathrm{CO}_{2}$ spectra. IV. Collisional line-mixing in infrared and Raman bands
}

\author{
J. Lamouroux, ${ }^{1}$ J.-M. Hartmann, ${ }^{1, a)}$ H. Tran, ${ }^{1}$ B. Lavorel, ${ }^{2}$ M. Snels, ${ }^{3}$ S. Stefani, ${ }^{4}$ \\ and G. Piccioni ${ }^{4}$ \\ ${ }^{1}$ Laboratoire Interuniversitaire des Systèmes Atmosphériques (LISA), CNRS UMR 7583, \\ Universités Paris Est Créteil et Paris Diderot, Institut Pierre-Simon Laplace, Université Paris Est Créteil, \\ 94010 Créteil Cedex, France \\ ${ }^{2}$ Laboratoire Interdisciplinaire Carnot de Bourgogne, Faculté des Sciences Mirande, \\ CNRS UMR 6303 et Université de Bourgogne, BP 47870, 21078 Dijon Cedex, France \\ ${ }^{3}$ ISAC-CNR, Via del Fosso del Cavaliere, 10000133 Rome, Italy \\ ${ }^{4}$ IAPS-INAF, Via del Fosso del Cavaliere, 10000133 Rome, Italy
}

(Received 3 May 2013; accepted 6 June 2013; published online 28 June 2013)

\begin{abstract}
Ab initio calculations of the shapes of pure $\mathrm{CO}_{2}$ infrared and Raman bands under (pressure) conditions for which line-mixing effects are important have been performed using requantized classical molecular dynamics simulations. This approach provides the autocorrelation functions of the dipole vector and isotropic polarizability whose Fourier-Laplace transforms yield the corresponding spectra. For that, the classical equations of dynamics are solved for each molecule among several millions treated as linear rigid rotors and interacting through an anisotropic intermolecular potential. Two of the approximations used in the previous studies have been corrected, allowing the consideration of line-mixing effects without use of any adjusted parameters. The comparisons between calculated and experimental spectra under various conditions of pressure and temperature demonstrate the quality of the theoretical model. This opens promising perspectives for first principle ab initio predictions of line-mixing effects in absorption and scattering spectra of various systems involving linear molecules. @ 2013 AIP Publishing LLC. [http://dx.doi.org/10.1063/1.4811518]
\end{abstract}

\section{INTRODUCTION}

The accurate modeling of pure $\mathrm{CO}_{2}$ spectra is essential for various applications, in particular for atmospheric remote sensing and radiative transfer studies. In a number of circumstances, the collision-induced transfers of rotational populations, and thus the exchanges of intensity within the spectrum (line-mixing (LM)), have to be taken into account in order to reach the required accuracies on the properties (pressure, temperature, etc.) retrieved from fits of remote sensed spectra. ${ }^{1}$ These LM effects yield an enhancement of the intense regions of the spectrum when the lines overlap significantly, a reduction of the band wings, and a filling of the gaps between doublets lines. Furthermore, the effects near the band or branch centers are more important when the collisionbroadened optical transitions overlap significantly. ${ }^{1}$ As a result, $\mathrm{LM}$ in $\mathrm{CO}_{2}$ has to be considered for the modeling of the infrared absorption/emission in the deep atmosphere of Venus (about $96.5 \%$ of $\mathrm{CO}_{2}$ ) where the pressure is high (up to 90 bar). Similarly, narrow infrared (e.g., Refs. 1-3) and Raman (e.g., Refs. 1 and 4-6) $\mathrm{CO}_{2} \mathrm{Q}$ branches are generally strongly affected by LM, even at low pressures, with consequences for remote sensing. ${ }^{1,7-9}$ Furthermore, although the effects of $\mathrm{LM}$ within and between $\mathrm{CO}_{2} \mathrm{P}$ and $\mathrm{R}$ branches are small at atmospheric pressure, they must be accurately

\footnotetext{
a) Author to whom correspondence should be addressed. Electronic mail: jean-michel.hartmann@lisa.u-pec.fr. Tel.: 33 (0)145176542. FAX: 33 (0) 145171564 .
}

modeled for a proper treatment of infrared atmospheric spectra. ${ }^{10,11}$

A quite exhaustive review of the models proposed to describe LM and of their applications to various molecular systems is given in Chap. IV of Ref. 1. Many studies have been devoted to mixtures involving $\mathrm{CO}_{2}$ with a few for the pure gas in the infrared at high pressure ${ }^{12-17}$ and Raman $\mathrm{Q}$ branches. ${ }^{4-6,18-20}$ Let us mention at this step that all LM models proposed up to now ${ }^{1}$ are partly empirical and that $a b$ initio predictions starting from the intermolecular potential have not yet been made. Finally note that the most used ${ }^{1}$ and accurate approach to describe LM effects is that based on the socalled Energy Corrected Sudden (ECS) approximation, with applications to pure $\mathrm{CO}_{2}$ in Refs. 6, 12,16, 19, and 20, and references therein. Despite that first principle quantum models have been proposed for the calculation of the relaxation matrix involved in the description of LM from the interaction potential, ${ }^{1}$ none are tractable nowadays (due to computer limitations) for $\mathrm{CO}_{2}-\mathrm{CO}_{2}$. Recently, a new approach was proposed for the $a b$ initio calculation of different absorption shape features without any adjusted parameters, based on Classical Molecular Dynamics Simulations (CMDS). It has been successively and successfully applied, for pure $\mathrm{CO}_{2}$, to the predictions of line broadening coefficients, ${ }^{21}$ of collisioninduced absorption, ${ }^{22}$ of the far wings of absorption and scattering bands, ${ }^{21,23}$ of individual line shapes, ${ }^{24}$ and to the collisional dissipation of laser-induced molecular alignment. ${ }^{25,26}$

In the present paper, properly requantized Classical Molecular Dynamics Simulations (rCMDS) are used for 
calculations of spectra under conditions which make linemixing effects important, a topic to which this approach had not yet been applied. We thus present thereafter the first $a b$ initio calculation of line-mixing for pure $\mathrm{CO}_{2}$ and compare their results with experiments. This is done, for various pressures and temperatures, for two infrared bands and the $2 v_{2}$ isotropic Raman Q branch. Section II describes the theoretical model and the modifications that have been made to properly take into account the line-mixing effects through rCMDS. This model is validated in Sec. III by comparisons with measured spectra and concluding remarks are given in Sec. IV.

\section{THEORETICAL MODEL AND DATA USED}

\section{A. Spectral shape}

As is well known, ${ }^{1}$ the absorption coefficient resulting from the interaction of a molecular intrinsic (i.e., not interaction induced ${ }^{1,27}$ ) dipole $\vec{d}$ with an electromagnetic field of angular frequency $\omega$ and wave vector $\vec{k}=(\omega / c) \vec{z}$ is given by

$$
F(\omega)=\operatorname{Re}\left\{\frac{1}{\pi} \int_{0}^{+\infty} \Phi(\omega, t) e^{-i \omega t} d t\right\},
$$

where $\Phi(\omega, t)$ is the autocorrelation function (ACF) of the dipole $\vec{d}(t)$

$$
\Phi(\omega, t)=\left\langle e^{-i \vec{k}(\omega) \cdot \vec{q}(t)} \vec{d}(t) \cdot \vec{d}(0) e^{+i \vec{k}(\omega) \cdot \vec{q}(0)}\right\rangle .
$$

In Eq. (2), $\langle\ldots\rangle$ denotes an average over the molecular system, $\vec{q}(t)$ is the molecule position, and the exponential terms result from the Doppler effect associated with the translational motion. This contribution is included in Eq. (2) for its completeness although the Doppler effect plays no role under the pressure conditions of the spectra considered in this study. Using the $\mathrm{ACF}$ of the isotropic polarizability $\alpha(0)$ [i.e., replacing $\vec{d}(t) \cdot \vec{d}(0)$ by $\alpha(t) \cdot \alpha(0)$ in Eq. (2)], Eq. (1) yields the isotropic Raman scattering spectrum.

\section{B. Classical molecular dynamics}

The approach used here for the calculation of $\Phi(\omega, t)$ is based on requantized classical molecular dynamics simulations. In these calculations, the force and torque applied to each molecule, treated as a linear and rigid rotor, by its surrounding sisters are computed classically at each time. ${ }^{21-24}$ This provides the center of mass position $\vec{q}_{m}(t)$ and velocity $\overrightarrow{\vec{q}}_{m}(t)$, the unit vector $\vec{u}_{m}(t)$ along the molecule axis, and the rotational angular momentum $\vec{\omega}_{m}(t)$ for each molecule $m$ at each time $t$. Note that if no requantization is made, the Fourier-Laplace transform of the relevant autocorrelation function leads to a continuous spectrum with no line structure. ${ }^{21,23}$ Most details of the method and of its implementation can be found in Ref. 24 and those therein. Nevertheless, the approach used in Refs. 24 and 28 suffered two limitations that were of no consequences for the studies made because the latter considered isolated lines. The first is that calculations were done for a single branch implicitly assuming identical $\mathrm{P}$ and $\mathrm{R}$ branches. This resulted from the fact that the information on the direction in which each molecule rotates was not used. The second is that the positions and intensities of the lines were incorrect since calculated for a strictly rigid rotor and neglecting centrifugal distortion. These inaccuracies must obviously be corrected for the prediction of the spectral shapes of entire bands or branches when the lines overlap significantly and are not isolated anymore.

\section{New requantization scheme}

In order to explain the new approach, let us start by considering dipole absorption for which we first focus on the calculation of the phase $\eta_{m}(t)$, such that $\vec{d}_{m}(t)$ $=\exp \left[i \eta_{m}(t)\right] \vec{d}_{m}(0)$. We then use the following four steps procedure for each molecule $m$. (i) We first determine the rotational quantum number from the correspondence principle for rotational energy. In other words, we find the even (for $\left.\mathrm{CO}_{2}\right)$ integer $J_{m}(t)$ for which $\frac{\hbar^{2}}{2 I} J_{m}(t)\left[J_{m}(t)+1\right]$ is the closest to $\frac{1}{2} I\left\|\vec{\omega}_{m}(t)\right\|^{2}$ ( $I$ being the $\mathrm{CO}_{2}$ molecule moment of inertia). This provides the value of $J$ for the lower state of the optical transition to which molecule $m$ contributes at time $t$. (ii) In order to now know which specific line $L_{m}(t)$ ( $L=\mathrm{R}$ or $\mathrm{P}$ ) is involved, we consider, following Ref. 29, the direction in which the molecule rotates. We hence look at the sign of the projection of the rotational angular momentum $\vec{\omega}_{m}(t)$ along the fixed axis $x$, deciding that positive and negative signs correspond to $L=\mathrm{R}$ and $L=\mathrm{P}$ branches, respectively. Due to isotropy, the choices of the projection axis and sign-to-branch correspondence are of no consequence. (iii) We can then calculate the true angular frequency corresponding to the line of initial rotational quantum number $J_{m}(t)$ in branch $L_{m}(t)$, from

$$
\omega_{L_{m}\left[J_{m}(t)\right]}=\omega_{V i b}+\omega_{R o t}^{U p}\left[J_{m}(t)+\Delta J_{m}(t)\right]-\omega_{R o t}^{L o w}\left[J_{m}(t)\right],
$$

where $\Delta J_{m}(t)=-1$ for $L_{m}(t)=P$ and $\Delta J_{m}(t)=+1$ for $L_{m}(t)$ $=R$. In Eq. (3), $\omega_{V i b}$ is the vibrational frequency for the considered band and $\omega_{R o t}^{L o w}(J)$ and $\omega_{R o t}^{U p}(J)$ are rotational energies in the lower and upper states. The latter are calculated from the proper rotational constants $B, D$, and $H$ through

$$
\begin{aligned}
\omega_{R o t}^{U p / L o w}(J)= & B^{U p / \text { Low }} J(J+1)-D^{U p / \text { Low }}[J(J+1)]^{2} \\
& +H^{U p / \text { Low }}[J(J+1)]^{3} .
\end{aligned}
$$

(iv) Finally, we calculate the phase $\eta_{m}(t)$ from

$$
\begin{aligned}
\eta_{m}(t) & =\int_{0}^{t} \omega_{L_{m}\left[J_{m}(t)\right]} \times \cos \left[\theta_{\mathrm{m}}(\mathrm{t})\right] d t \quad \text { with } \\
\cos \left[\theta_{\mathrm{m}}(\mathrm{t})\right] & =\left|\frac{\vec{\omega}_{m}(t) \cdot \vec{u}_{m}(0)}{\left\|\vec{\omega}_{m}(t)\right\|}\right|,
\end{aligned}
$$

where $\cos \left[\theta_{m}(t)\right]$ takes into account the angle between the plane in which the molecule rotates and the $\vec{d}_{m}(0)$ reference axis [recall that the dipole $\vec{d}(t)$ for the asymmetric stretching of $\mathrm{CO}_{2}$ is carried by the molecular axis $\left.\vec{u}(t)\right]$. Now that we know the phase and on which absorption line molecule $m$ absorbs at time $t$, the autocorrelation function is calculated from

$$
\begin{aligned}
& \Phi^{\text {absorption }}(\omega, t) \\
& =\frac{1}{N_{T}} \sum_{m=1, N_{T}}\left(d_{L_{m}\left[J_{m}(t)\right]}\right)^{2} e^{-i \vec{k}(\omega) \cdot\left[\vec{q}_{m}(t)-\vec{q}_{m}(0)\right]+i \eta_{m}(t)},
\end{aligned}
$$


where $N_{T}$ is the total number of molecules, and $d_{L(J)}$ is the dipole transition moment of the line of branch $\mathrm{L}$ of initial rotational quantum number $J$. Its value is directly obtained $^{1}$ from the line integrated intensity $S_{L(J)}$ through $d_{L(J)}=\sqrt{S_{L(J)} / p(J)}$ where $p(J)$ is the relative population of level J. These two quantities are easily calculated from the data provided in the used spectroscopic database (see Sec. II D).

For isotropic Raman scattering, the same procedure and equations are used except that $\cos \left[\theta_{\mathrm{m}}(\mathrm{t})\right]$ is removed from Eq. (5) (due to isotropy) as are $S_{L_{m}\left[J_{m}(t)\right]}$ and $\rho_{J_{m}(t)}$ in Eq. (6) [since this ratio is line independent]. Note that the considered $2 v_{2} \mathrm{Q}$ branch shows a significant spectral shift $\Delta_{V i b}$ and broadening $\Gamma_{V i b}(\mathrm{HWHM})$ due to vibrational effects (both linear vs density). ${ }^{18}$ Since these cannot be predicted by the rCMDS, they have been a posteriori introduced in the autocorrelation function. The latter is then given by

$$
\Phi^{\text {Raman }}(\omega, t)=\frac{1}{N_{T}} \sum_{m=1, N_{T}} e^{-i \vec{k}(\omega) \cdot\left[\vec{q}_{m}(t)-\vec{q}_{m}(0)\right]+i \eta_{m}(t)} e^{i \Delta_{v i b} t-\Gamma_{v i b} t}
$$

Note that the procedure described above, which provides the phase $\eta_{m}(t)$ and autocorrelation function $\Phi(\omega, t)$, is carried $a t$ each time step of the rCMDS. Also recall that the molecules' rotations are left unchanged as the requantization applied in Refs. 24 and 28 is not made anymore since it is now unnecessary.

\section{Data used and implementation}

Various input data are needed for calculations using the above described approach. The molecular parameters of $\mathrm{CO}_{2}$ and $\mathrm{CO}_{2}-\mathrm{CO}_{2}$ intermolecular potential ${ }^{30}$ are the same as those used in Ref. 24. $\omega_{V i b}$ and the $B, D$, and $H$ molecular constants in Eqs. (3) and (4) are from Ref. 31, while, for the infrared bands, the infrared spectroscopic parameters (line intensities and relative populations) were taken from Ref. 32 and converted to the temperature of the study. For the $2 v_{2}$ isotropic Raman Q branch, the density-normalized vibrational broadening and shifting coefficients $\gamma_{V i b}$ and $\delta_{V i b}$ were taken from Ref. 18.

Practical computations were made as described in Ref. 24 using the same initialisation procedure, time step, and algorithm. Furthermore, a dozen sets of about $10^{5}$ molecules were treated using the various cores of a parallel computer.

\section{RESULTS AND DISCUSSION}

This section is devoted to comparisons between measured spectra and those $a b$ initio calculated using the rCMDS approach. In addition to these, we also present spectra calculated neglecting LM in order to show the influence of this process. This was done independently of the rCMDS by simply summing the contributions of the individual lines, each of them having a Voigt profile. The input line intensities, populations, and line positions are the same as those used in the rCMDS while the broadening coefficients were taken from Ref. 16. Note that the same line widths are used for infrared and Raman lines, an approximation justified in Ref. 33.

\section{A. $3 v_{3}$ and $2 v_{1}+2 v_{2}+v_{3}$ infrared bands regions at room temperature}

For simplicity, rCMDS have been made only for the most intense band in each of the two spectral ranges considered in this work, i.e., the $3 v_{3}$ (around $6972.58 \mathrm{~cm}^{-1}$ ) and $2 v_{1}$ $+2 v_{2}+v_{3}$ (near $6347.85 \mathrm{~cm}^{-1}$ ) bands. This is due to the treatment of molecules as linear rotors which prohibits simulations when the bending mode of $\mathrm{CO}_{2}$ is involved. Hence, the relatively small contributions of minor bands (e.g., the first

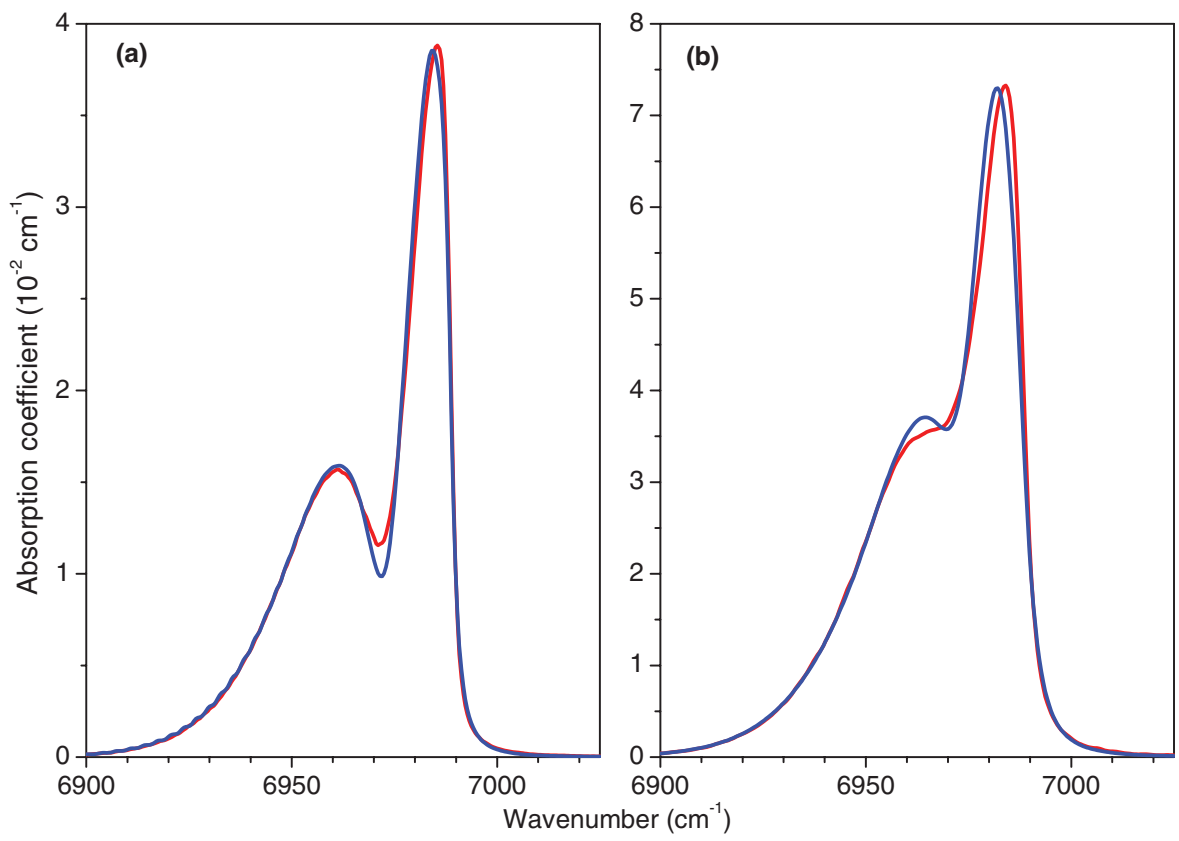

FIG. 1. Absorption coefficient of pure $\mathrm{CO}_{2}$ at $296 \mathrm{~K}$ due to the single $3 v_{3}$ band calculated with the present rCMDS model (red line) and the ECS approach of Ref. 16 (blue line) for the densities of (a) $22.65 \mathrm{Am}$ and (b) $51.28 \mathrm{Am}$. 


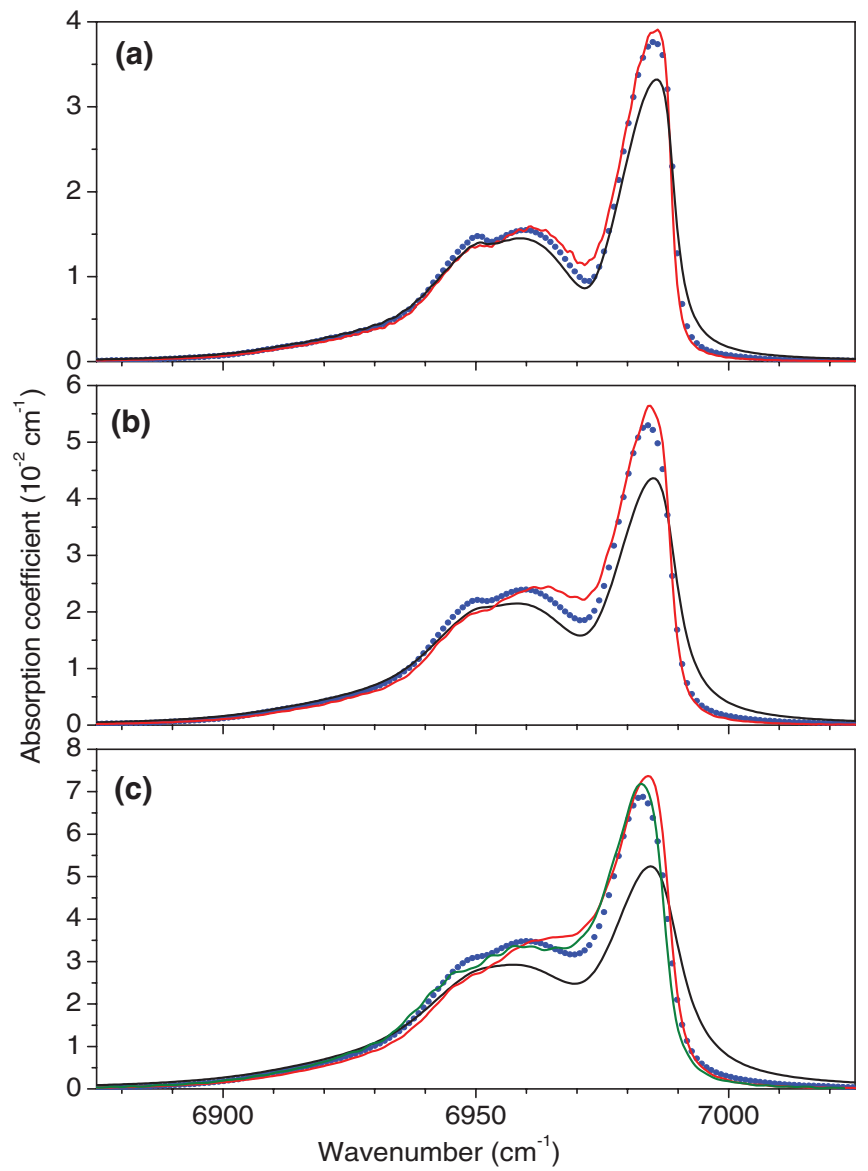

FIG. 2. Absorption coefficients of pure $\mathrm{CO}_{2}$ in the region of the $3 v_{3}$ band at $294 \mathrm{~K}$ for densities of (a) $22.7 \mathrm{Am}$, (b) $35.5 \mathrm{Am}$, and (c) $51.3 \mathrm{Am}$. The blue circles are measured values ${ }^{16}$ while the lines are calculated results obtained with the rCMDS model (red) and neglecting line-mixing (black). The olive curve in (c) has been obtained from rCMDS after the introduction of spectral shifts of opposite signs in the P and R branches (see text).

hot band $3 v_{3}+v_{2}-v_{2}$ ) have been calculated independently and added to the rCMDS results. This was done using the ECS model and tools of Ref. 16 which have been successfully validated in the same reference. Note that the comparisons, in Fig. 1, between ECS-calculated spectra and those obtained from rCMDS for the single $3 v_{3}$ band show the good agreement between these two completely independent approaches. A similar agreement is obtained for the $2 v_{1}+2 v_{2}+v_{3}$ band.

Calculated and measured ${ }^{16}$ spectra at $294 \mathrm{~K}$ in the region of the $3 v_{3}$ band for three gas densities are presented in Fig. 2. As expected from previous studies (see Refs. 16 and 17 and, more generally, Ref. 1), line-mixing has a significant influence that increases with pressure. The widths (respectively absorption peak) of the branches are overestimated (respectively underestimated) when this process is not taken into account with effects more pronounced in the $3 v_{3} \mathrm{R}$ branch due to the presence of a band-head around R(40). Figure 2 shows a satisfactory agreement, for all pressures, between the measured spectra and the rCMDS predictions, a result which validates the proposed model. However, some discrepancies remain, although they are much smaller than those obtained if LM is neglected. The most obvious is that the calculations underestimate the spectral distance between the maxima of the $\mathrm{P}$ and $\mathrm{R}$ branches and consequently overestimate the absorption

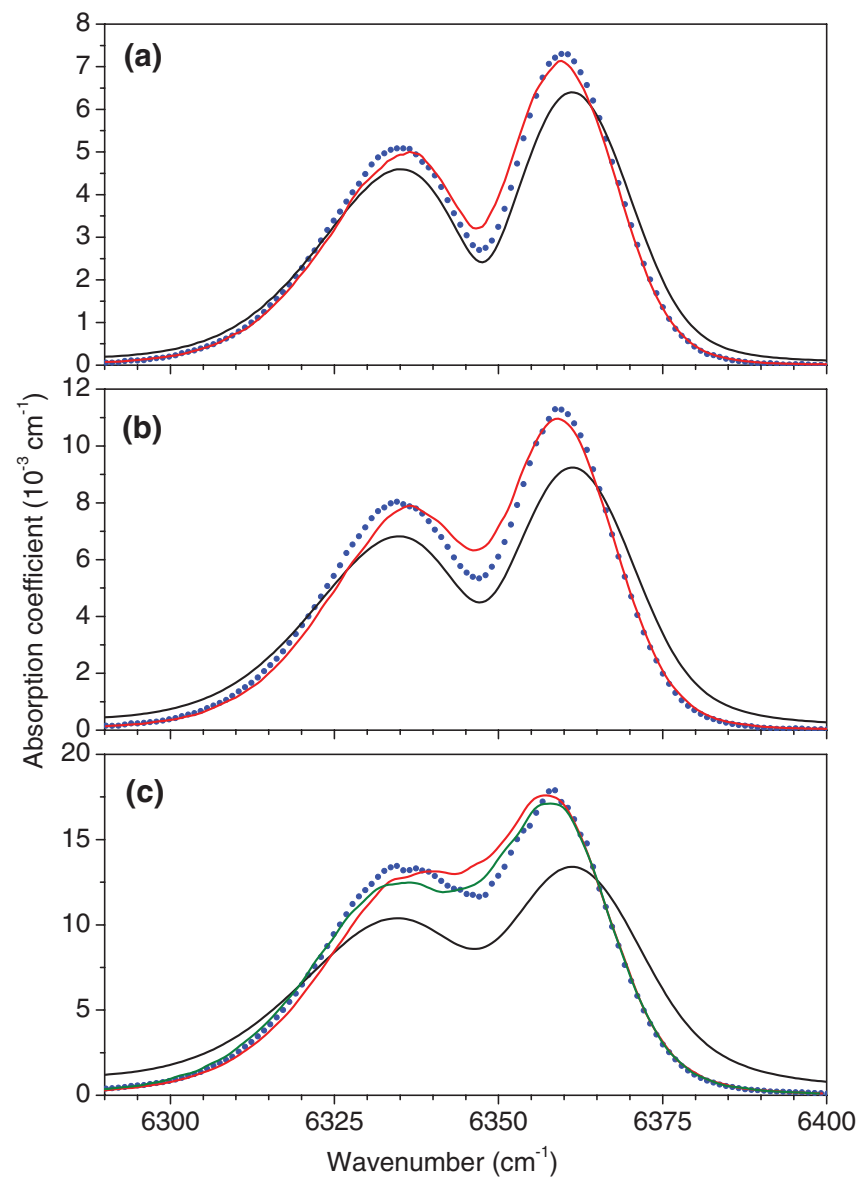

FIG. 3. Same as Fig. 2 but for the $2 v_{1}+2 v_{2}+v_{3}$ band region and densities of (a) 20.6 Am, (b) $33.0 \mathrm{Am}$, and (c) $56.7 \mathrm{Am}$.

near the band center. This discrepancy, which increases with the gas density, is likely due to the fact that the influence of the spectral shifts is not taken into account in the rCMDS. Indeed, as analyzed in Ref. 34, these lead, through the off-diagonal imaginary elements of the relaxation matrix, to spectral shifts of the $\mathrm{P}$ and $\mathrm{R}$ branches with respect to the band center of opposite signs. Hence the branches shift away from each other as the pressure increases, a process not taken into account in the rCMDS that likely explains the discrepancies in Fig. 2. In order to check this, and following Ref. 34, we have introduced spectral shifts independent of the transition but of opposite signs for $\mathrm{P}$ and $\mathrm{R}$ transitions by simply multiplying the autocorrelation functions in Eq. (6) by $\exp \left[i \int_{0}^{t} \Delta_{L_{m}(t)} d t\right]$ with $\Delta_{L_{m}(t)}=\Delta$ if $L_{m}(t)=\mathrm{R}$ and $\Delta_{L_{m}(t)}=-\Delta$ if $L_{m}(t)=\mathrm{P}$. As can be seen from the examples at elevated pressure in Figs. 2 and 3 , this greatly improves the results.

The results for the region of the $2 v_{1}+2 v_{2}+v_{3}$ band at $295 \mathrm{~K}$ are presented in Fig. 3 with measured spectra from Refs. 16 and 32. They lead to the same conclusions as those drawn above from the $3 v_{3}$ band region.

\section{B. $2 v_{2}$ isotropic Raman $Q$ branch at room temperature}

Comparisons between calculated spectra and measured ones $^{4}$ in the $2 v_{2}$ isotropic Raman Q branch of pure $\mathrm{CO}_{2}$ for three gas densities are shown in Fig. 4. The rCMDS are in striking agreement with the experimental spectra under 


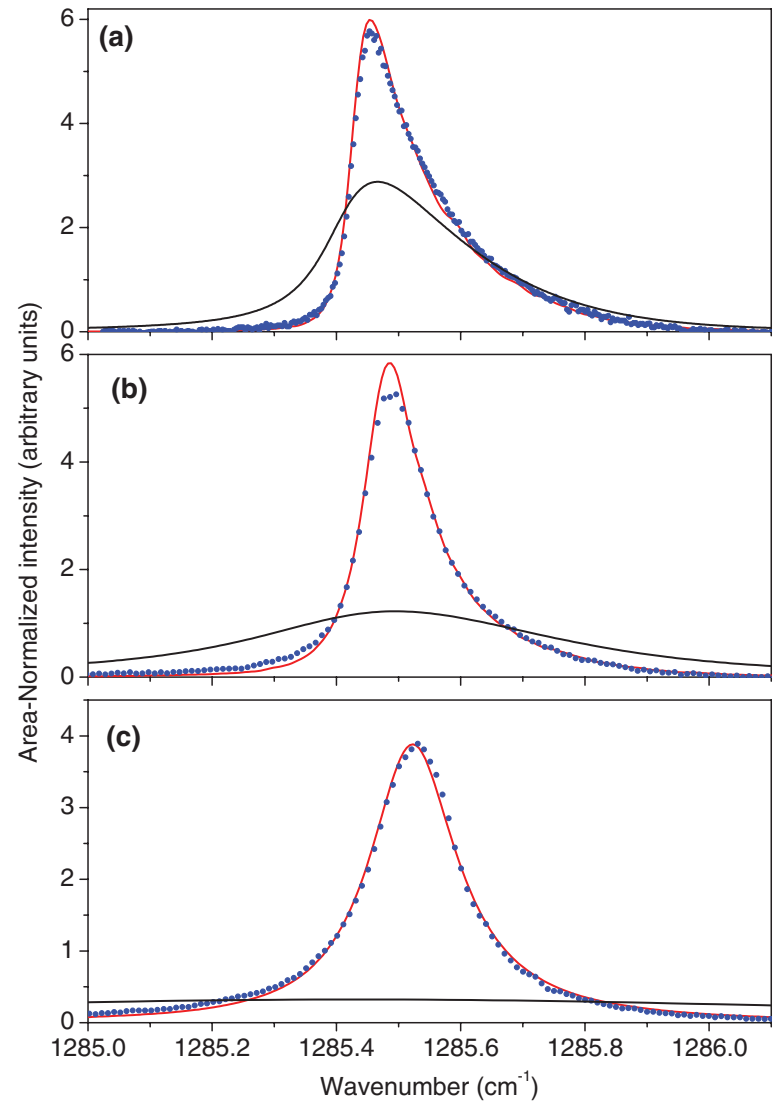

FIG. 4. Area-normalized intensities (in arbitrary units) in the $2 v_{2}$ isotropic Raman Q branch of pure $\mathrm{CO}_{2}$ at $295 \mathrm{~K}$ for the densities of (a) $0.5 \mathrm{Am}$, (b) $2.0 \mathrm{Am}$, and (c) $10.0 \mathrm{Am}$. The blue circles are measured values ${ }^{4}$ while the lines are calculated results obtained with the rCMDS model (red) and neglecting line-mixing (black).

conditions for which neglecting line-mixing leads to a considerable overestimation of the Q branch width. This last statement is explained by the well known fact ${ }^{1}$ that LM leads to a strong narrowing of isotropic Raman Q branches that can result in their collapse if no vibrational width is considered.

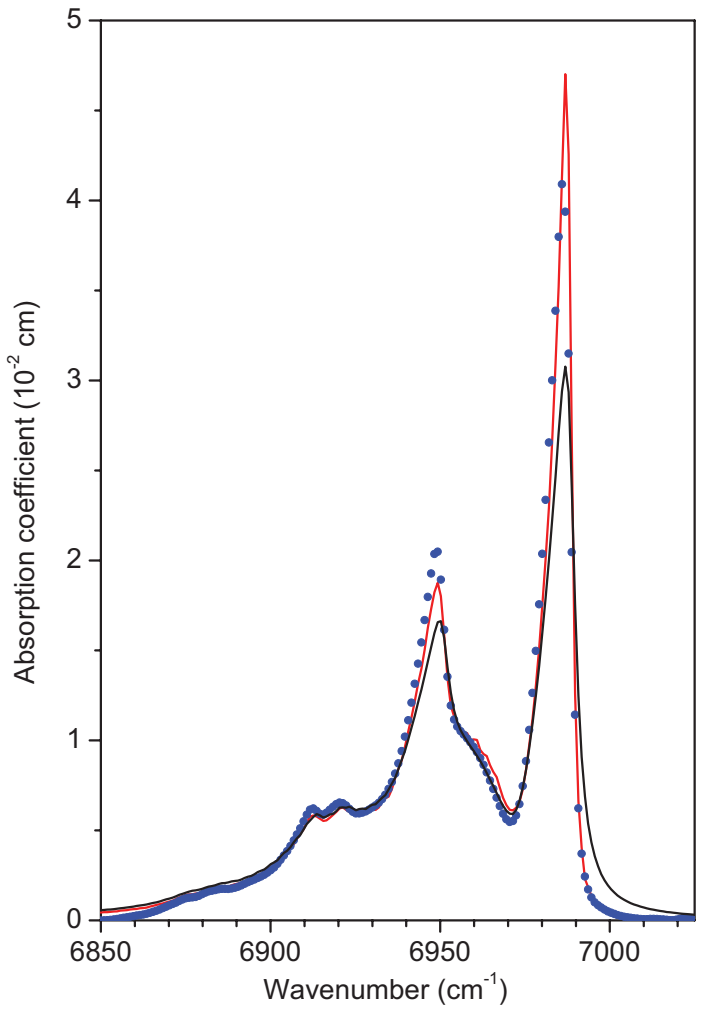

FIG. 5. Same as Fig. 2 but for $473 \mathrm{~K}$ and a density of $23.63 \mathrm{Am}$.

\section{Temperature effects}

For a final test of the rCMDS ability to describe the spectral effects of line-mixing, calculations were also made at higher temperatures. Comparisons between calculated spectra and those measured in the $3 v_{3}$ infrared region at $473 \mathrm{~K}^{16}$ and in the $2 v_{2}$ Raman $\mathrm{Q}$ branch at $700 \mathrm{~K}^{18}$ are presented in Figs. 5 and 6, respectively. For the $3 v_{3}$ band (Fig. 5), the significant contribution of several hot bands was, as mentioned above, taken into account using the ECS approach of

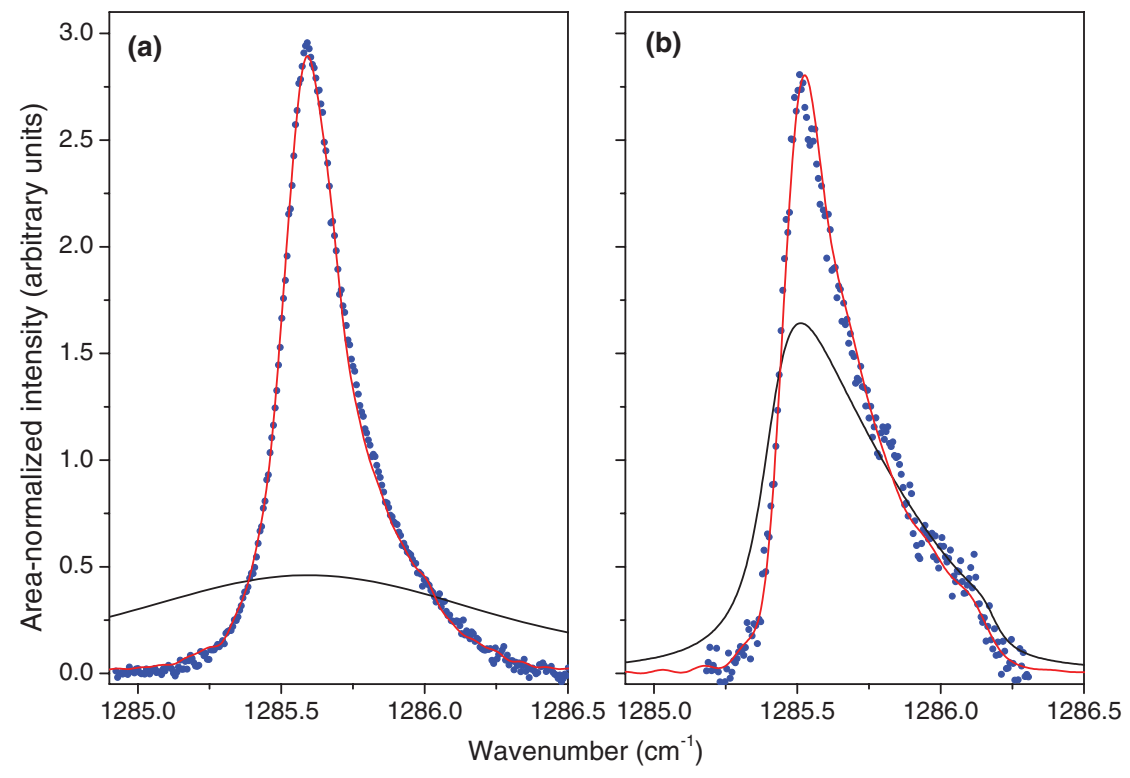

FIG. 6. Same as Fig. 4 but for $700 \mathrm{~K}$ and densities of (a) $5 \mathrm{Am}$ and (b) $1 \mathrm{Am}$. 
Ref. 16. Figures 5 and 6 again show that the rCMDS leads to much better agreement with experiments than the model neglecting line-mixing. Nevertheless, while very satisfactory results are obtained for the Raman Q branch, the quality of predictions in the infrared is poorer than at room temperature. The predicted $\mathrm{R}$ branch is too narrow, a finding for which we have no explanation that deserves further investigations. Finally note that, thanks to a requantization that now rigorously includes the line positions, the small "bump" near $1286.18 \mathrm{~cm}^{-1}$ in Fig. 6(b), due to the Q branch head at $\mathrm{J}=80$, is well reproduced.

\section{CONCLUSION}

In this paper, we present what are, to the best of our knowledge, the first $a b$ initio calculations of molecular spectra under conditions for which line-mixing significantly affects the spectral shape. The comparisons between predicted and measured infrared absorption and isotropic Raman scattering spectra demonstrate the quality of the proposed rCMDS model. Together with previous similar exercises for linebroadening, ${ }^{21}$ collision-induced absorption, ${ }^{22}$ wings of entire bands,${ }^{23}$ and isolated line-shapes, ${ }^{24}$ this demonstrates that rCMDS are a robust and flexible tool for the description of the consequences of inter-molecular collisions on $\mathrm{CO}_{2}$ spectra.

\section{ACKNOWLEDGMENTS}

J.L. thanks the French National Research Agency (ANR) for financial support through the Project ASGGRS (ANR-12PDOC-0012-01).

${ }^{1}$ J.-M. Hartmann, C. Boulet, and D. Robert, Collisional Effects on Molecular Spectra. Laboratory Experiments and Models, Consequences for Applications (Elsevier, Amsterdam, 2008).

${ }^{2}$ R. Rodrigues, K. W. Jucks, N. Lacome, Gh. Blanquet, J. Walrand, W. A. Traub, B. Khalil, R. Le Doucen, A. Valentin, C. Camy-Peyret, L. Bonamy, and J.-M. Hartmann, J. Quant. Spectrosc. Radiat. Transf. 61, 153 (1999).

${ }^{3}$ F. Niro, C. Boulet, J.-M. Hartmann, and E. Lellouch, J. Quant. Spectrosc. Radiat. Transf. 95, 483 (2005).

${ }^{4}$ B. Lavorel, G. Millot, R. Saint-Loup, H. Berger, L. Bonamy, J. Bonamy, and D. Robert, J. Chem. Phys. 93, 2176 (1990).

${ }^{5}$ B. Lavorel, G. Millot, R. Saint-Loup, H. Berger, L. Bonamy, J. Bonamy, and D. Robert, J. Chem. Phys. 93, 2185 (1990).

${ }^{6}$ B. Lavorel, G. Fanjoux, G. Millot, L. Bonamy, and F. Emond, J. Chem. Phys. 103, 9903 (1995).

${ }^{7}$ F. Niro, G. Brizzi, M. Carlotti, E. Papandrea, and M. Ridolfi, J. Quant. Spectrosc. Radiat. Transf. 103, 14 (2007).
${ }^{8}$ G. Millot, B. Lavorel, G. Fanjoux, and C. Wenger, Appl. Phys. B 56, 287 (1993).

${ }^{9}$ B. Lavorel, H. Tran, E. Hertz, O. Faucher, P. Joubert, M. Motzkus, T. Buckup, T. Lang, H. Skenderovi, G. Knopp, P. Beaud, and H. M. Frey, C. R. Phys. 5, 215 (2004).

${ }^{10}$ J.-M. Hartmann, H. Tran, and G. C. Toon, Atmos. Chem. Phys. 9, 7303 (2009).

${ }^{11}$ D. R. Thompson, D. C. Benner, L. R. Brown, D. Crisp, V. M. Devi, Y. Jiang, F. Oyafuso, D. Wunch, R. Castano, and C. E. Miller, J. Quant. Spectrosc. Radiat. Transf. 113, 2265 (2012).

${ }^{12}$ J.-M. Hartmann and C. Boulet, J. Chem. Phys. 94, 6406 (1991).

${ }^{13}$ J.-M. Hartmann and F. L'Haridon, J. Chem. Phys. 103, 6467 (1995).

${ }^{14}$ W. D. Gillespie, C. J. Meinrenken, W. R. Lempert, and R. B. Miles, J. Chem. Phys. 107, 5995 (1997).

${ }^{15}$ C. J. Meinrenken, W. D. Gillespie, S. Macheret, W. R. Lempert, and R. B. Miles, J. Chem. Phys. 106, 8299 (1997)

${ }^{16}$ H. Tran, C. Boulet, S. Stefani, M. Snels, and G. Piccioni, J. Quant. Spectrosc. Radiat. Transf. 112, 925 (2011).

${ }^{17}$ N. N. Fillippov, R. E. Asfin, T. N. Sinyakova, I. M. Grigoriev, T. M. Petrova, A. M. Solodov, A. A. Solodov, and J. Buldyreva, "Experimental and theoretical studies of $\mathrm{CO}_{2}$ spectra for planetary atmosphere modelling: Region $6000-10000 \mathrm{~cm}^{-1}$ and pressures up to 60 atm," Phys. Chem. Chem. Phys. (in press).

${ }^{18}$ B. Lavorel, G. Millot, G. Fanjoux, and R. Saint-Loup, J. Chem. Phys. 101, 174 (1994).

${ }^{19}$ L. Bonamy, J. Bonamy, D. Robert, A. Deroussiaux, and B. Lavorel, J. Quant. Spectrosc. Radiat. Transf. 57, 341 (1997).

${ }^{20}$ B. Lavorel, O. Faucher, M. Morgen, and R. Chaux, J. Raman Spectrosc. 31, 77 (2000).

${ }^{21}$ J.-M. Hartmann, C. Boulet, H. Tran, and M. T. Nguyen, J. Chem. Phys. 133, 144313 (2010).

${ }^{22}$ J.-M. Hartmann, C. Boulet, and D. Jacquemart, J. Chem. Phys. 134, 094316 (2011).

${ }^{23}$ J.-M. Hartmann and C. Boulet, J. Chem. Phys. 134, 184312 (2011).

${ }^{24}$ J.-M. Hartmann, H. Tran, N. H. Ngo, X. Landsheere, P. Chelin, Y. Lu, A.-W. Liu, S.-M. Hu, L. Gianfrani, G. Casa, A. Castrillo, M. Lepère, Q. Delière, M. Dhyne, and L. Fissiaux, Phys. Rev. A 87, 013403 (2013).

${ }^{25}$ J.-M. Hartmann and C. Boulet, J. Chem. Phys. 136, 184302 (2012).

${ }^{26}$ J.-M. Hartmann, C. Boulet, T. Vieillard, F. Chaussard, F. Billard, O. Faucher, and B. Lavorel, "Dissipation of alignment in $\mathrm{CO}_{2}$ gas: A comparison between $a b$ initio predictions and experiments," J. Chem. Phys. (submitted).

${ }^{27}$ L. Frommhold, Collision Induced Absorption in Gases (Cambridge University Press, Cambridge, 2006).

${ }^{28}$ J.-M. Hartmann, V. Sironneau, C. Boulet, T. Svensson, J. T. Hodges, and C. T. Xu, Phys. Rev. A 87, 032510 (2013).

${ }^{29}$ R. G. Gordon, J. Chem. Phys. 45, 1649 (1966).

${ }^{30}$ S. Bock, E. Bich, and E. Vogel, Chem. Phys. 257, 147 (2000).

${ }^{31}$ C. E. Miller and L. R. Brown, J. Mol. Spectrosc. 228, 329 (2004).

${ }^{32}$ J. Lamouroux, H. Tran, A. L. Laraia, R. R. Gamache, L. S. Rothman, I. E. Gordon, and J.-M. Hartmann, J. Quant. Spectrosc. Radiat. Transf. 111, 2321 (2010).

${ }^{33}$ L. Rosenmann, J.-M. Hartmann, M. Y. Perrin, and J. Taine, Appl. Opt. 27, 3902 (1988).

${ }^{34}$ R. Rodrigues, C. Boulet, L. Bonamy, and J.-M. Hartmann, J. Chem. Phys. 109, 3037 (1998). 